\title{
Comportamento de atributos químicos de um solo salino-sódico tratado com gesso e fósforo ${ }^{1}$
}

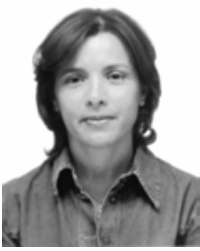

Adriana de F. M. Vital ${ }^{2}$, Rivaldo V. dos Santos ${ }^{3}$, Lourival F. Cavalcante ${ }^{4}$ \& Jacob S. Souto ${ }^{3}$

\author{
${ }^{1}$ Extraído da Dissertação de Mestrado apresentada pelo primeiro autor à UFPB \\ ${ }^{2}$ Frilance. E-mail: glendaleo@bol.com.br \\ ${ }^{3}$ DEF/UFCG, CEP 58700-970, Patos - PB, E-mail : rvital@cstr.ufcg.edu.br \\ ${ }^{4}$ Departamento de Solos e Engenharia Rural - Campus II - UFPB. CEP 58397-000, Areia - PB, E-mail: lofeca@cca.ufpb.br
}

Protocolo 16 - 3/2/2003 - Aprovado em 26/2/2004

\begin{abstract}
Resumo: Os solos degradados por sais e sódio, comuns nas áreas irrigadas da região semi-árida, apresentam atributos físicos e químicos desfavoráveis à agricultura. A baixa disponibilidade de nutrientes, como o fósforo, nesses solos é uma realidade. Desta forma o emprego de corretivos e o estudo da disponibilidade de fósforo nessas áreas constituem-se numa alternativa para reintegrar tais solos à produção agrícola. Conduziu-se um experimento para verificar o efeito da aplicação de quatro níveis de gesso (0,50, 100 e $200 \%$ da necessidade do gesso) e quatro dosagens de fósforo $\left(0,150,300\right.$ e $450 \mathrm{mg} \mathrm{dm}^{-3}$ ), em solo salino-sódico (Cambissolo - CE 4,2 dS $\mathrm{m}^{-1}$, PST 52, $\mathrm{pH} 6,3 \mathrm{e} \mathrm{CaCO}_{3} 65 \mathrm{~g} \mathrm{~kg}^{-1}$ ) de textura franco-argilosa (areia 18,8\%, silte 43,9\% e argila 37,3\%), do Perímetro Irrigado de Capoeira - São José do Bonfim, PB. Analisaram-se os parâmetros fósforo disponível, $\mathrm{Na}, \mathrm{Ca}, \mathrm{Mg}, \mathrm{pH}, \mathrm{PST}$ e $\mathrm{CE}$, cujos dados revelaram que o corretivo exerceu efeito significativo no aumento de cálcio e fósforo do solo e na redução da PST e pH. A aplicação de gesso e fósforo promoveu aumento significativo nos teores de $\mathrm{P}$ do solo e redução do $\mathrm{Na}$. Para as demais variáveis, o efeito dos tratamentos foi não significativo.
\end{abstract}

Palavras-chave: gesso, fósforo, salinidade, sodicidade

\section{Behavior of chemical parameters of a saline sodic soil treated with gypsum and phosphorus}

\begin{abstract}
The soils degraded by salts and sodium, common in the irrigated areas of the semi-arid region, present physical and chemical attributes unfavorable for the agriculture. The low availability of nutrients, like phosphorus, in these soils is a reality, therefore, employment of amendments and the study of availability of phosphorus in these areas constitute an alternative to reintegrate such soils to the agricultural production. An experiment was installed in a greenhouse to verify the effects of four levels of gypsum (0,50, 100 and $200 \%$ of gypsum requirement) and phosphorus $\left(0,150,300\right.$ and $450 \mathrm{mg} \mathrm{kg}^{-1}$ ), in a saline-sodic soil (Cambisol, EC $4.2 \mathrm{dS} \mathrm{m}^{-1}$; ESP 52, pH 6.3 and $\mathrm{CaCO}_{3} 65 \mathrm{~g} \mathrm{~kg}^{-1}$ ) of clay loam texture (sand 18,8\%, silt 43,9\% and loam 37,3\%) of the Irrigated Perimeter of Capoeira - São José do Bonfim - PB. Samples were collected to determine phosphorus availability, $\mathrm{Na}, \mathrm{Ca}, \mathrm{Mg}, \mathrm{pH}, \mathrm{PST}$ and EC. The analysis of the parameters showed that $\mathrm{P}$ availability, $\mathrm{Na}, \mathrm{Ca}, \mathrm{Mg}, \mathrm{pH}, \mathrm{PST}$ and $\mathrm{CE}$ showed that gypsum significantly increased $\mathrm{Ca}$ and $\mathrm{P}$ of the soil and decreased the $\mathrm{pH}$ and ESP. The application of gypsum and phosphorus increased $\mathrm{P}$ and decreased $\mathrm{Na}$. For the others variables the effect of the treatments was not significant.
\end{abstract}

Key words: gypsum, phosphorus, salinity, sodicity

\section{INTRODUÇÃO}

Um dos objetivos da agricultura é satisfazer as necessidades alimentícias da humanidade, que aumentam à medida que a população cresce. Segundo United Nations (1991) a população mundial é, atualmente, de cerca de 6 bilhões, havendo perspectiva de chegar a 8,5 bilhões no ano 2025. A incorporação de áreas afetadas por sais ao processo produtivo de alimentos tem relevante importância do ponto de vista social, econômico e ambiental (Gomes et al., 2000). Nesse contexto, a maximização da produtividade dos solos afetados por sais, através da aplicação de fertilizantes, é uma 
necessidade para que se supra, de forma adequada, as exigências alimentares da população.

A salinidade e a sodicidade do solo reduzem o potencial produtivo de grandes áreas do globo. Esses processos provocam a dispersão da argila, causando redução da penetração de água, ar e raízes e da capacidade de retenção de água disponível, dificultando uma agricultura adequada e eficiente (Levy et al., 1998; Shaw et al., 1998).

Para reabilitar os solos afetados por sais e incorporá-los à produção agrícola, é oportuno que seja promovida a remoção do sódio dos sítios de troca, usualmente proporcionada pela aplicação de gesso, seguida de lixiviação. A reação de troca entre o gesso e o sódio do solo depende do contato do corretivo com as partículas do solo e da taxa de remoção de sódio da solução do solo (Ilyas et al., 1997).

Apesar da literatura enfatizar um decréscimo na produção das culturas para um certo nível de salinidade, pode ocorrer aumento com a aplicação de fertilizantes (Luken, 1962; Lunin \& Gallatin, 1965). Desta forma, a melhoria nutricional pode ser uma alternativa para aumentar o crescimento e o desenvolvimento de culturas em ambientes estressados por sais (Feigin, 1985; Gibson, 1988; Soliman et al., 1994). Em muitos casos em que a água não é suficiente para lixiviar os sais abaixo da zona radicular, os agricultores devem modificar a seleção de culturas e o sistema de manejo, para minimizar os efeitos negativos sobre a produção e a rentabilidade.

Muitas pesquisas, sugerem que o fósforo pode exercer papel fundamental no sucesso do manejo de solos salinizados. As razões para explicar os resultados positivos do efeito da fertilização de fósforo nesses solos, não são ainda muito claras. Khalil et al. (1967) sugerem que as respostas positivas das plantas ao fósforo, sob condições salinas, podem ser devidas ao fato de que, sob tais condições, o crescimento radicular é limitado e uma elevada concentração de fósforo no solo pode compensar a demanda total da planta, neste nutriente.

As interações entre salinidade e nutrição de fósforo são bastante complexas, uma vez que são altamente dependentes da espécie ou da cultivar, do estádio de desenvolvimento, da composição e nível de salinidade e da concentração de fósforo no substrato; portanto, dependendo da planta selecionada e das condições do experimento, diferentes resultados podem ser obtidos (Grattan \& Grieve, 1998). Alguns trabalhos sugerem que a aplicação de gesso nesses solos reduz a disponibilidade de fósforo (Chhabra et al., 1981; Santos, 1995).

Este trabalho objetiva verificar o efeito da aplicação de níveis crescentes de gesso e fósforo sobre alguns atributos do solo e sobre a disponibilidade de fósforo no solo.

\section{MATERIAL E MÉTODOS}

A pesquisa foi desenvolvida no período de 25 de junho a 30 de setembro de 2001, em experimento conduzido em casade-vegetação localizada no Centro de Saúde e Tecnologia Rural da Universidade Federal de Campina Grande, em Patos, PB, cuja altitude é de $242 \mathrm{~m}$ e apresenta as seguintes coordenadas geográficas: latitude $17^{\circ} 01^{\prime} 28^{\prime \prime} \mathrm{S}$ e longitude $37^{\circ} 16^{\prime} 48^{\prime \prime} \mathrm{W}$. A temperatura média do local durante experimento, foi de $35^{\circ} \mathrm{C}$ (máxima) e $23^{\circ} \mathrm{C}$ (mínima) e a umidade relativa média do ar foi de $48 \%$.

Para o experimento, o solo foi coletado na profundidade de 0-30 cm, em área onde funcionou o Sistema de Irrigação Público de Capoeira, no município de São José do Bonfim, PB, semiárido paraibano, que possui uma área de $121,7 \mathrm{~km}^{2}$ e fica a 278 $\mathrm{m}$ acima do nível do mar, e apresenta as seguintes coordenadas geográficas: latitude norte $7^{\circ} 09^{\prime} 45^{\prime \prime}$ S e longitude $37^{\circ} 18^{\prime} 32^{\prime \prime}$ W. A pluviosidade média dos últimos dois anos no município foi de 808,3 mm (2000) e 517,3 mm (2001). O solo da área é um Cambissolo Háplico (EMBRAPA, 1999), de textura francoargilosa, salino-sódico, pouco profundo, moderadamente drenado, com declividade de 2,5\%. Após secado ao ar, o material de solo foi destorroado, peneirado (malha de $2 \mathrm{~mm}$ ) e homogeneizado e, a seguir, acondicionado em vasos plásticos com capacidade para $3 \mathrm{dm}^{3}$. A caracterização química inicial (Tabela 1) do solo foi efetuada segundo metodologia descrita em (EMBRAPA, 1997), exceto para CE1:5 e a CEes. A obtenção do extrato de saturação foi realizada segundo procedimento descrito em EMBRAPA (1997). Os teores de fósforo expressam o P ex-traível por Mehlich. $\left(\mathrm{H}_{2} \mathrm{SO}_{4} 0,025 \mathrm{~N}+\mathrm{HCl} 0,05 \mathrm{~N}\right)$. O cálcio, magnésio, sódio e potássio expressam os teores trocáveis mais solúveis, sendo os dois primeiros determinados por espectrofometria de absorção atômica e os dois últimos por fotometria de chama. A análise granulométrica foi determinada segundo Camargo et al. (1989). As variáveis estudadas foram submetidas à análise de variância e de regressão, utilizando-se o programa estatístico SANEST (Zonta et al., 1994).

Tabela 1. Alguns atributos químicos e físicos do solo estudado

\begin{tabular}{lc}
\hline \multicolumn{1}{c}{ Atributo } & Valor \\
\hline $\mathrm{pH}$ & 6,3 \\
$\mathrm{P}\left(\mathrm{mg} \mathrm{dm}^{-3}\right)$ & 1,0 \\
$\mathrm{Ca}^{2+}\left(\mathrm{cmol}_{\mathrm{c}} \mathrm{dm}^{-3}\right)$ & 6,7 \\
$\mathrm{Mg}^{2+}\left(\mathrm{cmol}_{\mathrm{c}} \mathrm{dm}^{-3}\right)$ & 8,3 \\
$\mathrm{Na}^{+}\left(\mathrm{cmol}_{\mathrm{c}} \mathrm{dm}^{-3}\right)$ & 16,64 \\
$\mathrm{~K}\left(\mathrm{cmol}_{\mathrm{c}} \mathrm{dm}^{-3}\right)$ & 0,05 \\
$\mathrm{CEes}\left(\mathrm{dS} \mathrm{m}^{-1}\right)$ & 34,0 \\
$\mathrm{CE} 1: 5\left(\mathrm{dS} \mathrm{m}^{-1}\right)$ & 4,2 \\
$\mathrm{PST}$ & 52 \\
$\mathrm{CaCO}\left(\mathrm{g} \mathrm{kg}^{-1}\right)$ & 65 \\
Areia $\left(\mathrm{g} \mathrm{kg}^{-1}\right)$ & 188 \\
Silte $\left(\mathrm{g} \mathrm{kg}^{-1}\right)$ & 439 \\
Argila $\left(\mathrm{g} \mathrm{kg}^{-1}\right)$ & 373 \\
Densidade do solo $\left(\mathrm{kg} \mathrm{dm}^{-3}\right)$ & 1,19 \\
Classificação textural & Franco-argilosa \\
Classificação & Salino-sódico \\
\hline
\end{tabular}

A princípio, realizou-se a aplicação de gesso no solo em quantidade calculada segundo Schoonover (1952). citado por Richards (1954), que recomenda a agitação de amostras do solo em solução saturada de $\mathrm{CaSO}_{4}\left(28 \mathrm{mmol} \mathrm{L}^{-1}\right)$, determinandose, ao se estabelecer o equilíbrio, o cálcio remanescente. A necessidade de gesso $(\mathrm{NG})$ foi então calculada pela fórmula: $\mathrm{NG}=\left[\mathrm{Ca}^{2+} \mathrm{SS}-\left(\mathrm{Ca}^{2+}+\mathrm{Mg}^{2+}\right)\right.$ remanescente na solução de equilibrio] x $2\left(\mathrm{cmol}_{\mathrm{c}} \mathrm{kg}^{-1}\right.$ solo $)$, onde $\mathrm{SS}=$ solução saturada com gesso. 
Neste trabalho o experimento foi arranjado em delineamento inteiramente casualizado, em esquema fatorial $(4 \mathrm{x} 4)$, correspondendo a 4 níveis de gesso $(0,50,100$ e $200 \%$ da NG estimada) e 4 níveis de fósforo $\left(0,150,300\right.$ e $\left.450 \mathrm{mg} \mathrm{dm}^{-3}\right)$, com três repetições, totalizando 48 unidades experimentais.

A primeira incubação foi realizada em delineamento inteiramente casualizado, com quatro níveis de gesso $(0,50$, 100 e $200 \%$ da NG estimada), em 12 repetições, totalizando 48 unidades experimentais. A NG estimada equivaleu a 3,3 g de gesso para cada $\mathrm{kg}$ de solo $\left(7,8 \mathrm{Mg} \mathrm{ha}^{-1}\right)$. O gesso foi incorporado ao material de solo previamente peneirado, sendo a mistura homogeneizada e mantida sob incubação por 20 dias, a uma umidade de $70 \%$ da capacidade de campo, mantida diariamente e se completando o volume de água estabelecido após pesagem dos vasos. Após vinte dias, os solos foram lavados pela adição de uma lâmina d'água extra, calculada em função do volume total de poros, correspondente a 1,1 L vaso $^{-1}$. Depois que os solos secaram, foram tomadas amostras de $50 \mathrm{~g}$ em cada vaso, nas quais se determinaram os valores de $\mathrm{P}$ extraível, $\mathrm{Na}, \mathrm{Ca}, \mathrm{Mg}$ e $\mathrm{K}$ trocáveis mais solúveis, $\mathrm{pH}$, conforme metodologia recomendada pela EMBRAPA (1997), e CE do extrato1:5.

Numa segunda etapa aplicou-se o fósforo, homogeneizando-o com todo o volume de solo, utilizando-se, como fonte, o fertilizante superfosfato simples, nos níveis $0,150,300$ e $450 \mathrm{mg} \mathrm{dm}^{-3}$ de fósforo, sob a forma de pó. O adubo foi triturado numa cuba; em seguida, o solo foi novamente incubado por mais um período de 20 dias, mantendo-se a umidade estabelecida para a primeira incubação. Ao término do período de incubação com o fertilizante fosfatado, foi retirada nova amostra de $50 \mathrm{~g}$ do solo para determinação dos teores disponíveis de P no solo, utilizando-se o extrator Mehlich-1 e teores trocáveis mais solúveis de $\mathrm{Na}, \mathrm{Ca}, \mathrm{Mg}$ e K (EMBRAPA, 1997).

\section{RESULTADOS E DISCUSSÃO}

\section{Efeito da aplicação do gesso nas propriedades químicas do solo}

Todas as variáveis analisadas sofreram variação ao longo do período do experimento, em relação à condição inicial do solo (Tabela 2). Foram observadas mudanças favoráveis nas propriedades do solo ao final do experimento.

A aplicação de gesso, no nível mais elevado, ocasionou ligeira acidificação do solo, porque na presença de gesso o

Tabela 2. Análise química do solo após a aplicação do gesso, incubação e lavagem*

\begin{tabular}{|c|c|c|c|c|c|c|c|}
\hline \multirow{2}{*}{ Variável } & \multirow{2}{*}{ Unidade } & \multicolumn{4}{|c|}{ Dosagens de Gesso (\%NG) } & \multirow{2}{*}{ Prob. $>$ F } & \multirow{2}{*}{ Efeito } \\
\hline & & 0 & 50 & 100 & 200 & & \\
\hline $\mathrm{Ca}$ & $\mathrm{cmol}_{\mathrm{c}} \mathrm{dm}^{-3}$ & 3,4 & 5,2 & 7,4 & 9,9 & $0,001 * *$ & $\mathrm{~L}$ \\
\hline $\mathrm{Mg}$ & $\mathrm{cmol}_{\mathrm{c}} \mathrm{dm}^{-3}$ & 3,5 & 3,1 & 2,4 & 2,5 & 0,055 & NS \\
\hline $\mathrm{Na}$ & $\mathrm{cmol}_{\mathrm{c}} \mathrm{dm}^{-3}$ & 3,42 & 2,18 & 3,82 & 2,53 & 0,549 & NS \\
\hline $\mathrm{P}$ & $\mathrm{mg} \mathrm{dm}{ }^{-3}$ & 1,0 & 1,0 & 2,0 & 4,0 & $0,000 * *$ & $\mathrm{~L}$ \\
\hline K & $\mathrm{cmol}_{\mathrm{c}} \mathrm{dm}^{-3}$ & 0,10 & 0,11 & 0,13 & 0,12 & 0,057 & NS \\
\hline $\mathrm{pH}$ & - & 6,5 & 6,2 & 6,2 & 6,0 & $0,047^{*}$ & $\mathrm{~L}$ \\
\hline $\mathrm{CE}_{1: 5}$ & $\mathrm{dS} \mathrm{m}^{-1}$ & 3,0 & 3,2 & 1,9 & 3,9 & 0,513 & NS \\
\hline PST & $\%$ & 33 & 20 & 28 & 17 & $0,045^{*}$ & $\mathrm{~L}$ \\
\hline $\mathrm{Ca} / \mathrm{Mg}$ & - & $1 / 1$ & $2 / 1$ & $3 / 1$ & $4 / 1$ & - & \\
\hline
\end{tabular}

*ALS após a lixiviação dos sais; NS e L não significativo e linear cálcio substitui o sódio trocável e, através da lixiviação pela água de irrigação, são removidos os íons $\mathrm{Na}^{+} \mathrm{e} \mathrm{OH}$, causando decréscimo no $\mathrm{pH}$ e no teor de sódio do solo. A relação é expressa pela equação $\mathrm{pH}=-0,0022 \mathrm{x}+6,42\left(\mathrm{R}^{2}=0,85^{*}\right)$. Esta redução do $\mathrm{pH}$ se deve também, em parte, à retirada dos cátions $\left(\mathrm{Ca}^{2+}, \mathrm{Mg}^{2+}, \mathrm{K}^{+}\right.$e $\left.\mathrm{Na}^{+}\right)$do complexo sortivo e sua substituição por íons $\mathrm{H}^{+}$, provenientes da dissociação iônica da água, bem como pelo efeito do corretivo que, ao aumentar a concentração de eletrólitos na solução do solo, tende a diminuir o pH. Reduções no pH em virtude da aplicação do gesso em solos salino-sódicos têm sido observadas por diversos pesquisadores (Gupta \& Goi, 1992; Santos, 1995; Qadir et al., 1996; Verma \& Sharma, 2000; Duran et al., 2000; Gomes et al., 2000 ), os quais comentam que tais reduções ocorreram também em razão do cultivo desses solos.

A concentração de sais, avaliada através dos valores de condutividade elétrica determinados em extratos solo:água de $1: 5$, tendeu a reduzir-se em função do aumento dos níveis de gesso aplicados, exceto para o nível máximo, cuja causa pode

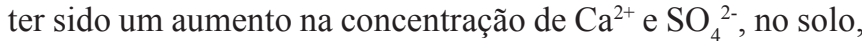
derivado do gesso, quando se aplicaram $200 \%$ da $\mathrm{NG}$, em razão de uma solubilização menor do gesso, e de menor lixiviação de sais solúveis, fato observado em teste de laboratório quando se constatou que os valores da condutividade elétrica aumentam com a elevação da concentração de gesso contida na solução. Esse fato foi também observado por Cavalcante (1984), Holanda (1996) e Gomes et al. (2000). Na ausência de gesso, a condutividade elétrica apresentou redução em comparação com o valor inicial, devido à lixiviação dos sais após a lavagem do solo.

Todos os tratamentos foram eficientes na redução da percentagem de saturação por sódio. A relação é expressa pela equação PST $=-0,0629 x+30 R^{2}=0,54 *$. Os maiores decréscimos na PST podem ser atribuídos ao aumento da concentração de cálcio na solução do solo, proveniente do gesso, que originou o deslocamento do sódio adsorvido. A elevada fração do $\mathrm{CaCO}_{3}$ nativo nesse solo $\left(65 \mathrm{~g} \mathrm{~kg}^{-1}\right)$, também pode ter contribuído para o suprimento de cálcio, após sua solubilização, com a redução do $\mathrm{pH}$.

Verificou-se decréscimo da PST já apenas com a lavagem (testemunha) o que pode ser explicado pela "diluição", como demonstrado por Reeve \& Bower (1960): no sistema solo-água, onde cátions mono e divalentes na solução estão em equilíbrio com os cátions adsorvidos, a adição de água ao sistema altera as condições de equilíbrio. Esta diluição da solução do solo favorece a adsorção de cátions divalentes como $\mathrm{Ca}^{2+}$ à custa de cátions monovalentes, como $\mathrm{Na}^{+}$. O inverso é verdadeiro quando a solução do solo é concentrada devido à evapotranspiração (Qadir et al., 1996).

Os valores de $\mathrm{Ca}^{2+}, \mathrm{Mg}^{2+}, \mathrm{K}^{+} \mathrm{e} \mathrm{Na}$, (Tabela 2), correspondem aos teores trocáveis mais solúveis, enquanto os teores de cálcio sofreram grande variação. No tratamento controle, a simples lavagem contribuiu para sua lixiviação e após, o período de lixiviação dos sais, depois da aplicação do corretivo químico, a concentração de $\mathrm{Ca}^{2+}$ aumentou de 3,4 para 9,9 $\mathrm{cmol}_{\mathrm{c}} \mathrm{dm}^{-3}$, evidenciando a capacidade do gesso, apesar de sua baixa solubilidade, para elevar a concentração de $\mathrm{Ca}^{2+}$ trocáveis, quando aplicado em solo salino-sódico. 
Com relação ao magnésio, seus valores decresceram consideravelmente, à exceção do nível mais elevado de gesso. Pesquisas apontam para uma tendência a um deslocamento maior do magnésio após a aplicação do gesso em solos sódicos (Chaudhry \& Warkentin, 1968), justificado pelo fato de que um percentual do $\mathrm{Ca}^{2+}$ aplicado substitui o $\mathrm{Mg}^{2+}$ trocável do solo (Armstrong \& Tanton, 1992).

A Tabela 2 mostra também um aumento acentuado na relação $\mathrm{Ca} / \mathrm{Mg}$ após a aplicação do corretivo, a qual variou de 1:1 para 4:1 na omissão e $200 \%$ da NG, respectivamente; tal elevação, no entanto, é benéfica, pois a maioria das culturas apresentam um crescimento vegetal mais adequada quando o solo tem relação $\mathrm{Ca} / \mathrm{Mg}$ de 4 .

Os teores de sódio trocável mais solúvel apresentaram uma tendência à redução nos vários tratamentos, embora sem indicar significância estatística; todavia, a concentração de sódio do solo foi consideravelmente reduzida $\left(16,6\right.$ para $\left.3,42 \mathrm{cmol}_{\mathrm{c}} \mathrm{dm}^{-3}\right)$ logo após a lavagem do solo.

Para o potássio, a tendência foi de pequena elevação da concentração em relação ao solo original e independente do tratamento devido, possivelmente, a solubilização do K do solo.

Os teores de fósforo apresentaram tendência de aumento com os níveis crescentes de gesso (Figura 1), devido ao fato de que o gesso agrícola utilizado apresentou $12,9 \mathrm{mg} \mathrm{P} \mathrm{kg}^{-1}$, diferentemente do resultado de outras pesquisas (Gupta et al., 1990; Santos, 1995; Batra et al., 1997) mas se assemelham aos de Cavalcante et al. (1998) que estudaram o efeito do gesso agrícola sobre um solo irrigado sem problemas de sais, e de Lucena (1986) que trabalhou com um solo alcalino tratado com fosfogesso.

\section{Efeito do fósforo nas propriedades químicas do solo}

A aplicação de dosagens crescentes de gesso proporcionou significativo aumento nos teores de $\mathrm{P}$ disponível do solo, apenas quando se aplicaram 300 e $450 \mathrm{mg} \mathrm{P} \mathrm{kg}{ }^{-1}$ (Tabela 3). Possivelmente, a maior concentração de sulfato presente no solo, nas maiores dosagens de gesso, proporcionou também maior dessorção do fósforo. Verifica-se, ainda, que a aplicação de doses crescentes de P aumentou significativamente seus teores disponíveis no solo, independentemente do gesso aplicado.

Para o sódio, constata-se uma significativa redução de sua concentração com o aumento das dosagens de $\mathrm{P}$ e de gesso, exceção para os seus níveis mais elevados (Tabela 4), o que se atribui a uma substituição maior do $\mathrm{Na}^{+}$trocável do solo pelo

Tabela 3. Teores de P extraível no solo após a incubação nas diferentes dosagens de gesso e fósforo*

\begin{tabular}{|c|c|c|c|c|c|c|}
\hline \multirow{3}{*}{$\begin{array}{c}\mathrm{P} \\
\mathrm{mg} \mathrm{dm}\end{array}$} & \multicolumn{4}{|c|}{ Dosagens de gesso $(\% \mathrm{NG})$} & \multirow{3}{*}{ Prob. $>$ F } & \multirow{3}{*}{ Efeito } \\
\hline & 0 & 50 & 100 & 200 & & \\
\hline & \multicolumn{4}{|c|}{$\mathrm{mg} \mathrm{dm}{ }^{-3}$} & & \\
\hline 0 & 1 & 2 & 2 & 2 & 0,877 & NS \\
\hline 150 & 55 & 53 & 50 & 48 & 0,216 & NS \\
\hline 300 & 110 & 106 & 136 & 150 & 0,000 & $\mathrm{~L}$ \\
\hline 450 & 193 & 246 & 193 & 260 & 0,020 & $\mathrm{Q}$ \\
\hline Prob. $>$ F & 0,001 & 0,000 & 0,000 & 0,000 & & \\
\hline Efeito & Q & $\mathrm{Q}$ & $\mathrm{L}$ & $\mathrm{Q}$ & & \\
\hline
\end{tabular}

A.

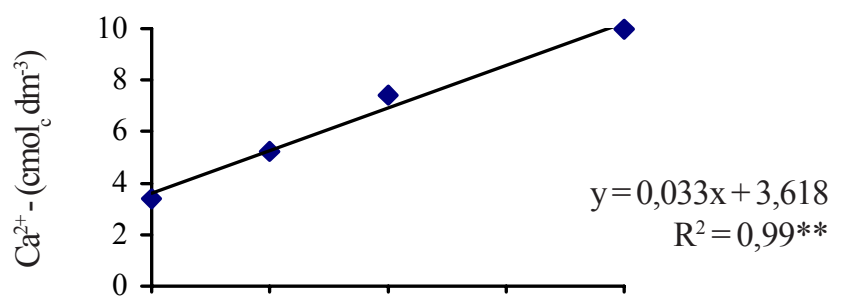

B.

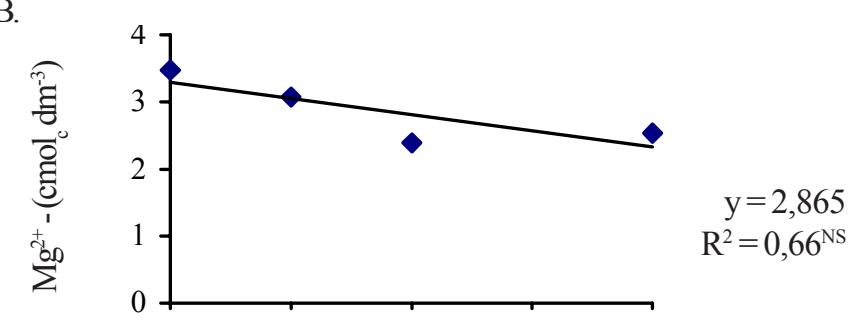

C.

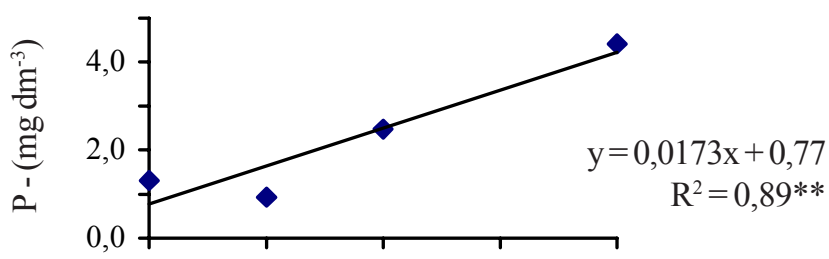

D.

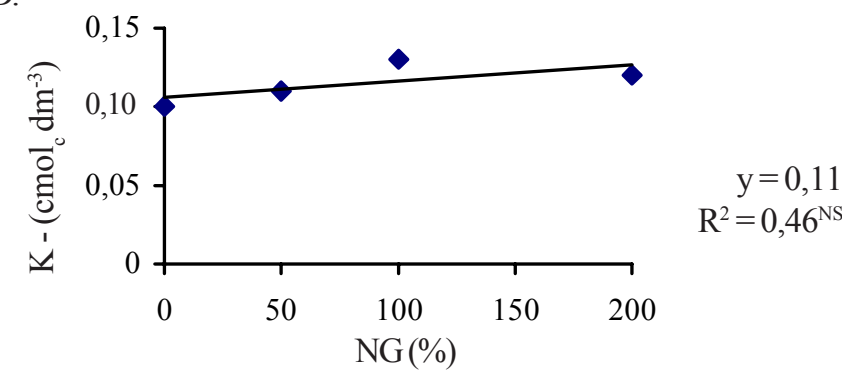

Figura 1. Efeito da aplicação de gesso (NG) sobre os teores de $\mathrm{Ca}$ (A), $\mathrm{Mg}(\mathrm{B}), \mathrm{P}(\mathrm{C})$ e $\mathrm{K}$ (D) no solo

Tabela 4. Teores de Na trocável no solo após incubação, nas dosagens de gesso e fósforo*

\begin{tabular}{|c|c|c|c|c|c|c|}
\hline \multirow{3}{*}{$\begin{array}{c}\mathrm{P} \\
\mathrm{mg} \mathrm{dm}\end{array}$} & \multicolumn{4}{|c|}{ Dosagens de gesso $(\% \mathrm{NG})$} & \multirow{3}{*}{ Prob. $>$ F } & \multirow{3}{*}{ Efeito } \\
\hline & 0 & 50 & 100 & 200 & & \\
\hline & \multicolumn{4}{|c|}{$\mathrm{cmol}_{\mathrm{c}} \mathrm{dm}^{-3}$} & & \\
\hline 0 & 5,16 & 3,82 & 1,65 & 2,36 & 0,048 & Q \\
\hline 150 & 3,79 & 9,49 & 2,36 & 2,36 & 0,001 & $\mathrm{~L}$ \\
\hline 300 & 3,75 & 3,75 & 5,48 & 2,55 & 0,044 & Q \\
\hline 450 & 2,76 & 2,73 & 2,08 & 3,16 & 0,745 & NS \\
\hline Prob. $>$ F & 0,041 & 0,001 & 0,011 & 0,527 & & \\
\hline Efeito & $\mathrm{L}$ & $\mathrm{Q}$ & $\mathrm{Q}$ & NS & & \\
\hline
\end{tabular}

$\mathrm{Ca}^{2+}$ derivado tanto do gesso presente no fertilizante super simples (40-50\%) quanto do gesso aplicado como corretivo.

Independentemente do $\mathrm{P}$ aplicado, a concentração de $\mathrm{Ca}^{2+}$ no solo aumentou consideravelmente com os níveis crescentes de gesso aplicados e o $\mathrm{Mg}^{2+}$ trocável apresentou tendência de queda, (Tabela 5), semelhantemente ao período da primeira incubação quando o $\mathrm{Mg}^{2+}$ do solo reduziu-se de 3,5 para 2,5 cmolc $\mathrm{dm}^{-3}$. 
Tabela 5. Teores de Ca e Mg extraíveis do solo após incubação com gesso, independentemente das doses de fósforo*

\begin{tabular}{ccc}
\hline \multirow{2}{*}{ Dosagens de gesso $(\% \mathrm{NG})$} & $\mathrm{Ca}^{2+}$ & $\mathrm{Mg}^{2+}$ \\
\cline { 2 - 3 } & \multicolumn{2}{c}{$\mathrm{cmol}_{\mathrm{c}} \mathrm{dm}^{-3}$} \\
50 & 6,4 & 3,4 \\
100 & 7,8 & 3,4 \\
200 & 8,4 & 3,2 \\
Prob. $>\mathrm{F}$ & 12,0 & 1,7 \\
Efeito & $0,001^{* *}$ & $0,007^{* *}$ \\
\hline
\end{tabular}

De igual forma, a aplicação de $\mathrm{P}$, independentemente do gesso, também propiciou a mesma tendência de redução, (Tabela 6), o que se deve ao fato de que, independentemente da mineralogia do solo, o cátion $\mathrm{Ca}^{2+}$ tem preferência pelos pontos de troca das argilas em relação ao $\mathrm{Mg}^{2+} \mathrm{e}$, desta forma, ocorre substituição deste pelo $\mathrm{Ca}^{2+}$, ocasionando uma lixiviação maior do $\mathrm{Mg}^{2+}$ devido à formação de $\mathrm{MgSO}_{4}$ nas dosagens mais altas de gesso.

Tabela 6. Teores de Ca e Mg trocáveis do solo após a incubação com fósforo, independentemente das dosagens de gesso

\begin{tabular}{ccc}
\hline \multirow{2}{*}{$\mathrm{P}\left(\mathrm{mg} \mathrm{dm}^{-3}\right)$} & $\mathrm{Ca}^{2+}$ & $\mathrm{Mg}^{2+}$ \\
\cline { 2 - 3 } & \multicolumn{2}{c}{$\mathrm{cmol}_{\mathrm{c}} \mathrm{dm}^{-3}$} \\
\hline 0 & 4,2 & 2,88 \\
300 & 5,7 & 3,79 \\
450 & 7,2 & 3,02 \\
Prob. $>\mathrm{F}$ & 7,6 & 2,10 \\
Efeito & 0,000 & 0,000 \\
\hline *L & $\mathrm{L}$ & $\mathrm{Q}$ \\
\hline
\end{tabular}

A representação gráfica do efeito do gesso e do fósforo, nos teores de $\mathrm{P}$ disponível e de sódio trocável, encontra-se nas Figuras 2 (A e B) e 3, respectivamente. As equações de regressão para o gráfico $\mathrm{A}$ são $\mathrm{y}=0,0011 \mathrm{X}^{2}+0,0257 \mathrm{X}+206,77$ $\left(R^{2}=0,40^{*}\right), y=0,2266 X+106,02\left(R^{2}=0,85^{*}\right), y=51,8$ e $y=1,7$, para $\mathrm{P}_{450}, \mathrm{P}_{300}, \mathrm{P}_{150}$ e $\mathrm{P}_{00}$, respectivamente. Para o gráfico $\mathrm{B}$ as equações são $\mathrm{y}=0,0007 \mathrm{X}^{2}+0,267 \mathrm{X}+0,295\left(\mathrm{R}^{2}=0,99 * *\right), \mathrm{y}=$ $0,4404 \mathrm{X}-3,59\left(\mathrm{R}^{2}=0,98 * *\right), \mathrm{y}=0,001 \mathrm{X}^{2}+0,08 \mathrm{X}+0,225\left(\mathrm{R}^{2}=\right.$ $0,99 * *)$ e $y=0,0003 X^{2}+0,276 X+2,41\left(R^{2}=0,99 * *\right)$, para $G_{200}$, $\mathrm{G}_{50}, \mathrm{G}_{00}$ e $\mathrm{G}_{100}$, respectivamente. Verifica-se que o fósforo extraível do solo aumentou significativamente com a aplicação das doses de gesso, mas apenas nos maiores níveis de fósforo, 300 e $450 \mathrm{mg} \mathrm{kg}^{-1}$. Isso se deve a maior saturação das argilas do solo com o cátion cálcio, que passam a reter mais fósforo. Segundo Tisdale et al. (1984) essa ação do cálcio é possível quando o $\mathrm{pH}$ do solo é ligeiramente inferior a 6,5 , como no solo em estudo, cujos $\mathrm{pH}$ nas maiores doses de gesso variaram de 6,0 para 6,2 (Tabela 2 ). Tal comportamento não foi verificado na omissão de fósforo devido sua reduzida concentração ( $1 \mathrm{mg} \mathrm{kg}^{-1}$ ), como indica a Tabela 1. Pela Figura 2B constata-se que independentemente dos níveis de gesso, a aplicação de doses de fósforo crescentes nos solos salinizados aumentou significativamente seus teores disponíveis nos solos. As equações de regressão para o Figura 3 são $y=0,0009 X^{2}+0,0507 X+5,36\left(R^{2}=0,92 *\right), y=$
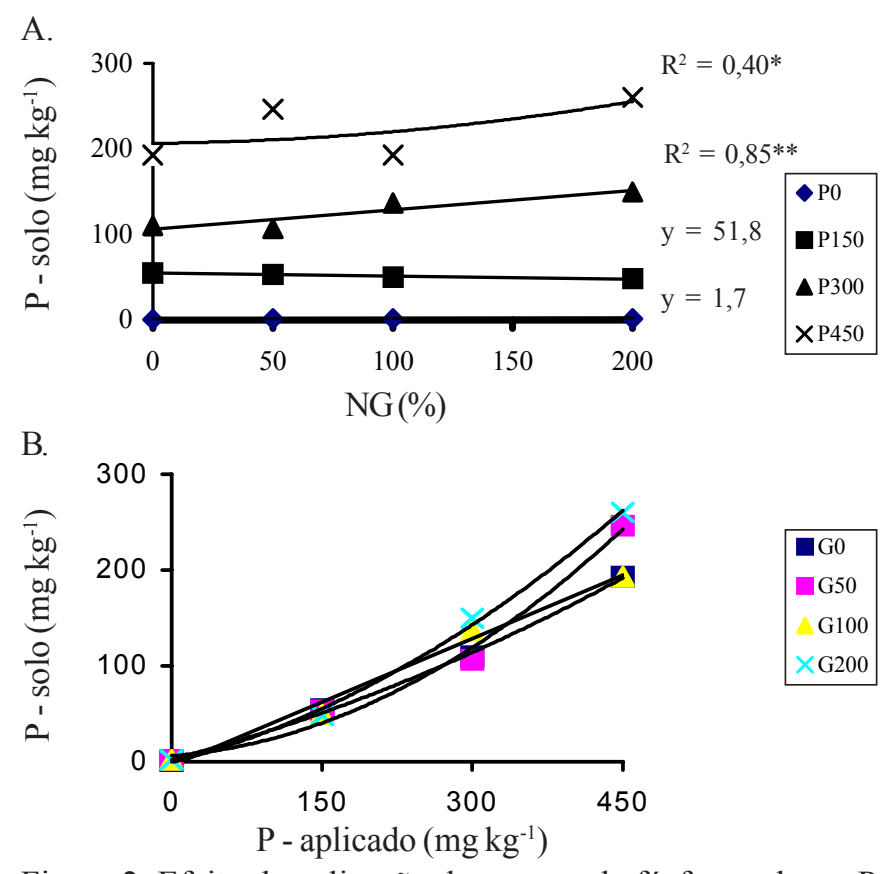

Figura 2. Efeito da aplicação de gesso e de fósforo sobre o $\mathrm{P}$ extraível nas diferentes dosagens de gesso (A) e de fósforo (B) aplicados ao solo

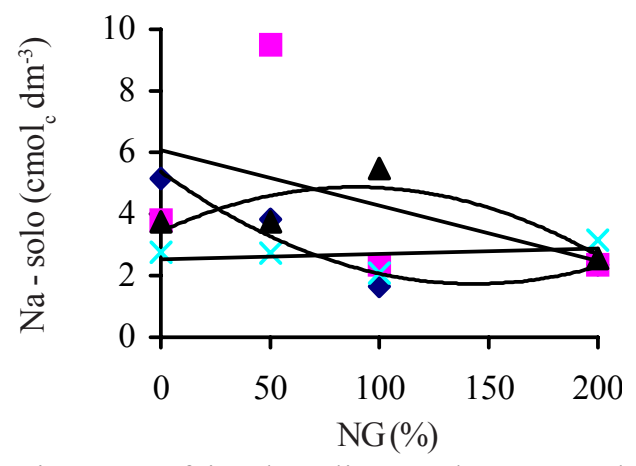

Figura 3. Efeito da aplicação de gesso e de fósforosobre os teores de sódio do solo nas diferentes de fósforo aplicados ao solo

$-0,0179 \mathrm{X}+6,07\left(\mathrm{R}^{2}=0,20 * *\right), \mathrm{y}=0,0002 \mathrm{X}^{2}+0,0322 \mathrm{X}+3,43\left(\mathrm{R}^{2}=\right.$ $0,72 *)$ e $\mathrm{y}=2,68$ nas doses $\mathrm{P}_{00}, \mathrm{P}_{150}, \mathrm{P}_{300}$ e $\mathrm{P}_{450}$, respectivamente. Observa-se que o gesso reduziu significativamente os teores de sódio trocável do solo em todas as doses de fósforo, exceto a $450 \mathrm{mg} \mathrm{kg}^{-1}$, o que confirma também para as condições do solo em estudo a capacidade desse corretivo em neutralizar o sódio trocável, facilitando sua remoção de solos degradados por excesso de sódio.

\section{CONCLUSÕES}

1. A aplicação do corretivo gesso em solo salino-sódico altera favoravelmente alguns atributos químicos do solo, dentre os quais, aumenta a disponibilidade de fósforo e de cálcio do solo e promove redução do $\mathrm{pH}$ e dos teores de sódio.

2. Recomenda-se a aplicação de gesso em solos salinosódico, que exige adubação fosfatada, como alternativa para elevar-se os teores de fósforo disponíveis. 
3. Independentemente da aplicação de corretivo, o fósforo aplicado em solos salino-sódicos apresentam uma elevada disponibilidade.

\section{AGRADECIMENTOS}

À CAPES, pela concessão da bolsa de estudos durante o Mestrado ao primeiro autor.

Á professora Sandra Barreto Queiroz (CCA) pelo auxílio na descrição do perfil.

\section{LITERATURA CITADA}

Armstrong, A.S.B.; Tanton, J.W. Gypsum applications to aggregated saline-sodic clay topsoil. Journal of Soil Science, Oxford, v.43, p.249-260, 1992.

Batra, L.; Kumar, A.; Manna, M.C.; Chhabra, R. Microbiological and chemical amelioration of alkaline soil by growing Karnal Grass and gypsum application. Experimental Agriculture, Cambridge, v.33,p.389-397, 1997.

Camargo, O.A. de; Moniz, A.; Jorge, J.A. Valadares, J.M.A.S. Métodos de análise química, mineralógica e física do solo do Instituto Agronômico de Campinas. Campinas: Instituto Agronômo, 1986. 94p. Boletim Técnico 106

Cavalcante, L.F. Efeito do fosfogesso em solos salinizados da Paraíba cultivados com feijão vigna (Vigna unguiculata L. Walp). Piracicaba : ESALQ/USP, 1984. 94p. Tese Doutorado

Cavalcante, L.F.; Vieira, J.E.; Oliveira, F.A.; Santos, C.J.O.; Souza, C.C. Teores de sódio, cálcio e fósforo no solo e absorvidos por plantas de vigna em um solo irrigado tratado com gesso agrícola I: Solo normal quanto à salinidade. Anais Curso de Pós-graduação em Manejo de Solo e Água, Areia, v.20, p.2331, 1998

Chaudhry, G.H.; Warkentin, B.P. Studies on exchange of sodium from soils by leaching with calcium sulfate. Soil Science, Baltimore, v.105, n.3, p.191-197, 1968.

Chhabra, R.; Abrol, I.P.; Singh, M.V. Dynamics of phosphorus during reclamation of sodic soils. Soil Science, Baltimore, v.132, n.5, p.319-324, 1981.

Duran, R.; Garcia, J.; Amaya, R. Amendment evaluation for the reclamation of sodic soils in the Cesar Valley. Suelos Ecuatoriales, Quito, v.30, n.1, p.21-28, 2000.

EMBRAPA. Centro Nacional de Pesquisa de Solos (Rio de Janeiro, RJ). Manual de métodos de análise de solo. 2 ed. rev. atual. Rio de Janeiro: EMBRAPA/CNPS, 1997. 212 p. Documentos 1

EMBRAPA. Centro Nacional de Pesquisa de Solos (Rio de Janeiro, RJ). Sistema brasileiro de classificação de solos. 2 ed. rev. atual. Rio de Janeiro: EMBRAPA Solos, 1999. 412 p.

Feigin, A. Fertilization management of crops irrigated with saline water. Plant and Soil, Dordrecht, v.89, p.285-299, 1985.

Gibson, T.S. Carbohydrate metabolism and phosphorus/salinity interactions in wheat (Triticum aestivum L.). Plant and Soil, Dordrecht, v.111, p.25-35, 1988.
Gomes, E.M.; Gheyi, H.R.; Silva, E.F. de F.E. Melhorias nas propriedades químicas de um solo salino-sódico e rendimento de arroz, sob diferentes tratamentos. Revista Brasileira de Engenharia Agrícola e Ambiental, Campina Grande, v.4, n.3, p.355-361, 2000.

Grattan, S.R.; Grieve, C.M. Salinity-mineral nutrient relations in horticultural crops. Scientia Horticulture, Amsterdam, v.78, n.1-4, p.127-157, 1998.

Gupta, J.P.; Goi, A.T.R. Effect of gypsum applications on physicchemical characteristics of sodic soil and crop yield of rice (Oryza sativa) and wheat (Triticum aestivum). Indian Journal of Agronomy, New Delhi, v. 37, n.4, p. 812-814, 1992.

Gupta, R.K.; Singh, R.R.; Tanji, K.K. Phosphorus release in sodium ion dominated soils. Soil Science Society of American Journal, Madison, v.54, p.1254-1260, 1990.

Holanda, J.S. de. Manejo de solo salino-sódico na região do Baixo Açu-RN. Piracicaba : ESALQ/USP, 1996. 83 p. Tese Doutorado

Ilyas, M.; Qureshi, R.H; Qadir, M.A. Chemical changes in a saline-sodic soil after gypsum application and cropping. Soil Technology, New York, v.10, p.247-260, 1997.

Khalil, M.A.; Amar, F.; Elgabaly, M.M. A salinity-fertility study on corn and cotton. Soil Science Society of America Proceedings, Madison, v.31, p.683-686, 1967.

Levy, G.J.; Shainberg, I.; Miller, W.P. Physical properties of sodic soils. In.: Summer, M.E.; Naidu, R. (eds.), Sodic soils: Distribution, properties, management and environmental consequences. New York: Oxford University Press, 1998. p.77-94.

Lucena, E.R. Efeito de aplicação de fosfogesso em um solo salino-sódico. Piracicaba : ESALQ/USP, 1986. 94p. Dissertação Mestrado

Luken, H. Saline soils under dryland agriculture in Southeast Saskatchewan (Canada) and possibilities for their improvement. Plant and Soil, Dordrecht, v.17, n.1, p.26-48, 1962.

Lunin, J.; Gallatin, M.H. Salinity-fertility interactions in relation to the growth and composition of beans. II. Varying levels of N and P. Agronomy Journal, Madison, v. p. 1965.

Qadir, M.; Qureshi, R.H.; Ahmad, N. Reclamation of a salinesodic soil by gypsum and Leptochloa fusca. Geoderma, Amsterdam, v.74, p.207-217, 1996.

Reeve, R.C.; Bower, C.A. Use of high-salt waters as a flocculant and source of divalent cations for reclaiming sodic soils. Soil Science, Baltimore, v.90, p.139-144, 1960.

Santos, R.V. dos. Correção de um solo salino-sódico e absorção de nutrientes pelo feijoeiro vigna (Vigna unguiculata $(\mathrm{L}$.) WALD.). Piracicaba: ESALQ/USP, 1995. 120 p. Tese Doutorado

Schoonover, W.R. Examination of soils for alkali. Berkeley: University of California Extension Service. 1952. Mimeographed

Shaw, R.J.; Coughlan, K.J.; Bell, L.C. Root zone sodicity. In.: Summer, M.E.; Naidu, R. (eds.), Sodic soils: Distribution, properties, management and environmental consequences. New York: Oxford University Press, 1998. p.95-106. 
A. de F. M. Vital et al.

Soliman, M.S.; Shalabi, H.G.; Campbell, W.F. Interaction of salinity, nitrogen and phosphorus fertilization on wheat. Journal of Plant Nutrition, New York, v.17, n.7, p.1163-1173, 1994.

Tisdale, S. L. , Nelson, W. L.; Beaton, J. D. Soil fertility and fertilizers. New York, Macmillan Publishing Company, 1984. $754 \mathrm{p}$.

United Nations. World population prospects. New York. United Nations, 1991, 607p. Populations Studies, 120.
Verma, S.K.; Sharma, O.P. Growth and yield of cotton (Gossypium hirsutum) in black alkali soils as influenced by methods of gypsum application. Indian Journal of Agriculture Science, New Delhi, v.70, n.11, p.791-793, 2000.

Zonta, E.P.; Machado, A.A.; Silveira Júnior, P. Sistemas de análise estatística para microcomputadores (SANEST). Pelotas, UFPel - Departamento de Solos. 1994. 35p. 